\title{
ANSWER : une opération de sciences participatives dans le domaine de l'eau
}

\author{
Jean-Michel TANGUY \\ Ministère de l'environnement, de l'énergie et de la mer - direction de la recherche et de l'innovation
}

\begin{abstract}
RÉSUMÉ. - Le ministère de l'écologie, du développement durable et de l'énergie a lancé une opération de sciences participatives dans le domaine de l'eau : ANSWER. Cette opération qui associe grand public et scientifiques a pour objectif d'améliorer les connaissances dans le domaine de l'eau. Le grand public est invité à photographier, filmer et conduire des expérimentations en nature en tant que témoins de phénomènes hydrauliquement intéressants, comme la propagation - déformation des houles, les ondes dans les canaux, les courants dans les rivières en crue, les effets des ouvrages en mer ou en rivière, les déformations des fonds des rivières mobiles... Ces vidéos peuvent concerner des événements en période normale ou pendant des situations extrêmes : inondations ou submersions marines ; ils constituent alors des témoignages essentiels pour les retours d'expérience.

Les documents produits peuvent être commentés par les scientifiques mais également être utilisés pour des besoins de publication. L'opération ANSWER repose sur l'outil collaboratif WIKHYDRO qui est le wiki du ministère de l'écologie dans le domaine de l'eau. Ouvert sur Internet, il contient 1600 pages, 300 vidéos.

Les premiers retours d'ANSWER sont encourageants et quelques vidéos illustrent des phénomènes qui ont aiguisé la curiosité des scientifiques. L'une des prolongations de la démarche est de pouvoir utiliser les vidéos prises pendant des crues ou des submersions marines pour quantifier certaines données comme les niveaux d'eau ou encore la vitesse du courant.

ANSWER répond à une évolution de notre société connectée, qui utilise les nouvelles technologies d'information et de communication (TIC) : internet, smartphones, réseaux sociaux, capteurs intelligents... . Le citoyen est ainsi acteur de son territoire en fournissant des données géoréférencées. Leur exploitation en quantité et en qualité peut ainsi contribuer à améliorer les politiques publiques de prévention, de prévision et de résilience des territoires.
\end{abstract}

Mots-clés : sciences participatives, hydraulique, vidéos techniques, wiki eau, NTIC

\section{ANSWER: a scientific participatory initiative in the field of water}

\begin{abstract}
The French ministry of Ecology, Sustainable Development and Energy decided to launch a scientist participatory initiative in the field of water. This initiative brings the general public and scientist community together, with the aim to improve the knowledge in the field of water. The general public is invited to take pictures, film and conduct field experiments as witness of hydraulically interesting processes as propagation - deformation of surface waves in rivers as well as in oceans, river bed evolutions... The corresponding videos can refer to common or exceptional events: they will also constitute precious first hand documents.

The documents produced can be commented by scientists and utilized for publishing purposes.

ANSWER initiative is based on the collaborative tool WIKHYDRO, which is the wiki of the ministry of Ecology, Sustainable Development and Energy in the water field. Opened on Internet, it contains 1600 pages, 300 videos.

The first feedback some months after the launch is positive and encouraging and some videos illustrating some physical phenomena have already raised the curiosity of the scientists. The follow-up of the initiative is to use the videos recorded during floods or storm surges to quantify some data as water levels or water velocities.

ANSWER is a response to our connected society, which uses new technologies of information and communication technologies (ICT): Internet, smartphones, social networks, smart sensors... The citizen, who provides geo-referenced data, becomes a major actor of his territory. The qualitative and quantitative big data mining can contribute to improve public policies in the fields of prevention, forecasting and resilience of territories.
\end{abstract}

Key-words: participatory science, hydraulics, technical videos, water wiki, ICT

\section{INTRODUCTION}

Avec l'émergence du Web 2.0, les technologies et les réseaux traditionnels d'information et de communication ont été bousculés. Le boom du numérique et l'apparition des réseaux sociaux ont permis de décupler l'utilisation des supports de communication (photographies, animations, vidéos, etc.) mais aussi d'accélérer leur vitesse de diffusion (messageries instantanées, tweets, textos, etc.).

De leur côté, les citoyens sont devenus producteurs et partageurs de données, notamment par le biais des nouvelles technologies comme les smartphones et les appareils photos numériques. 
En situation de crise, les réseaux sociaux fournissent des photos et des vidéos de première main sur le déroulement des catastrophes et permettent d'affiner l'intervention des secours.

L'association de ces nouvelles pratiques et technologies a fait naître diverses initiatives basées sur le principe de la participation, de la collaboration.

Depuis le début des années 2000, de nombreux projets ont été lancés avec l'objectif de favoriser l'expression de cette expertise citoyenne en mettant à contribution des communautés autour de problèmes précis (community sourcing). Parmi les cas les plus marquants sont ceux du Médaillé Fields Tim Gowers qui a posté un problème à résoudre sur Internet (Polymath Projects ${ }^{1}$ ), du joueur d'échec Kasparov qui a joué contre le monde ${ }^{2}$, du dénombrement des galaxies (Galaxy Zoo ${ }^{3}$ par le Sloan Digital Sky Survey), etc.

En France, de nombreuses initiatives existent déjà comme les programmes de données de suivi tel que Vigie Nature ${ }^{4}$ qui propose à chacun de contribuer à la recherche en découvrant la biodiversité qui nous entoure, les programmes d'inventaires de la Ligue de Protection des Oiseaux, les programmes d'observation phénologique du CNRS, etc., mais aucune démarche n'a encore été lancée à notre connaissance dans le domaine de l'eau.

Toutes ces initiatives ont fait avancer les connaissances et ont produit des résultats collatéraux (sérendipité).

\section{ANSWER : UNE DÉMARCHE DE SCIENCES PARTICIPATIVES}

Le MEDDE a décidé de lancer la démarche de sciences participatives : ANSWER $^{5}$ dont l'objectif est de faire collaborer public et scientifiques afin d'améliorer les connaissances dans le domaine de l'eau sous toutes ses formes (inondations, submersions marines, houles, marée, évolution des fonds ou pollution des eaux).

Le public est invité à photographier, filmer, conduire des expérimentations en nature, produire de la donnée sur des phénomènes liés à l'eau, etc. Ces contributions, dont un accusé de réception est fourni à l'émetteur, sont postées après validation et avis scientifique des organismes du GISHEDD $^{6}$ sur le site WIKHYDRO $^{7}$, le wiki du ministère dans le domaine de l'eau. Cette plate-forme abrite quelque 1600 pages et 300 vidéos à caractères techniques et scientifiques. Il s'agit d'un réservoir de première importance pour réceptionner et diffuser sur internet la production d'ANSWER, à savoir les vidéos produites par le public et les travaux des scientifiques qui incluent des vidéos réalisées en laboratoires des organismes de recherche.

La cible du projet ANSWER est constituée du grand public au travers de la mise à disposition des photos et des vidéos, le public averti (professeurs, étudiants, professionnels de l'eau), des organismes de formation initiale et continue (universités, écoles, lycées, collèges).

\footnotetext{
1. http://polymathprojects.org/

2. http://www.chessgames.com

3. http://www.galaxyzoo.org/

4. http://vigienature.mnhn.fr

5. http://wikhydro.developpement-durable.gouv.fr/index.php/ANSWER_-$\mathrm{Pr} \% \mathrm{C} 3 \%$ A9sentation de $1 \% 27$ op\%C3\%A9ration

6. Groupement d'Intérêt Scientifique Hydraulique pour l'Environnement et le Développement Durable qui regroupe 15 laboratoires universitaires, 7 organismes du réseau scientifique et technique, 9 organismes privés.

7. www.wikhydro.developpement-durable.gouv.fr
}

Un premier test de validité a été conduit auprès d'un public resserré et de premiers résultats encourageants ont pu être enregistrés : une trentaine de vidéos ont été collectées sur des processus assez étonnants (Biarritz $\left.{ }^{8}, \underline{\text { Soliton }}^{9}\right)$.

Différents modes de valorisation de ces informations en provenance du public sont envisagés : publication dans des revues, réalisation d'un ouvrage en réalité augmentée où les vidéos, assorties d'avis scientifiques, seront accessibles par des flashcodes à partir de smartphones. Le public pourra prendre connaissance sur le site collaboratif de l'ensemble de ces contributions et ainsi améliorer ses connaissances dans le domaine de l'eau.

Le diagramme ci-dessus (Figure 1) illustre les flux entre producteurs et utilisateurs et identifie les modes de valorisation des productions.

\section{LA PLATE-FORME COLLABORATIVE WIKHYDRO}

WIKHYDRO est un outil collaboratif de partage et de diffusion de la connaissance dans le domaine de l'eau mis en place par le MEDDE. Il est ouvert aux contributions des organismes du réseau scientifique et technique du ministère, des services déconcentrés de l'Etat et des services techniques de collectivités, et de professionnels du domaine. Il est libre d'accès en consultation sur Internet.

De nombreuses connaissances techniques de haut niveau, détenues par les agents du ministère et par les collectivités territoriales sont souvent peu formalisées et diffusées vers l'extérieur. Or notre société rend nécessaire la diffusion de l'information qui, à son tour, peut devenir une nouvelle source de richesse et contribuer à améliorer l'éducation du public, afin de le rendre plus apte à faire face aux grands défis de notre société.

WIKHYDRO a été conçu pour répondre à la nécessité de valoriser les organismes du ministère, mais également ses agents, et de construire une communauté de spécialistes de l'eau. La production de l'ensemble des organismes est de premier plan, en quantité comme en qualité, et répond à un besoin d'informations très souvent innovantes.

WIKHYDRO a vocation à rassembler les contributions de professionnels qui interviennent dans des domaines tels que : - la gestion des régimes hydrologiques et hydrauliques des eaux et de leur qualité, la police de l'eau, la prévention et la prévision des inondations ;

- la gestion du domaine fluvial ou maritime ;

- les évolutions morphodynamiques des milieux, telles que l'évolution des fonds des rivières ou des littoraux ;

- les activités touristiques des milieux côtiers et fluviaux, incluant les problèmes de sécurité des populations, la qualité des eaux aussi bien souterraines que superficielles, ou les éventuels conflits d'usage ;

- la gestion et la protection des ressources en eau, le traitement et l'évacuation des eaux pluviales et des eaux usées ; - le maintien de la biodiversité et la préservation ou la restauration des écosystèmes.

8. http://wikhydro.developpement-durable.gouv.fr/index.php/ANSWER Plage_de_Biarritz,_France

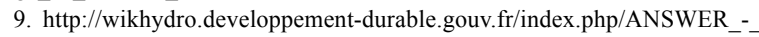
Soliton,_Mus\%C3\%A9um_d\%27histoire_naturelle_du_Havre_-_essais_ physiques 


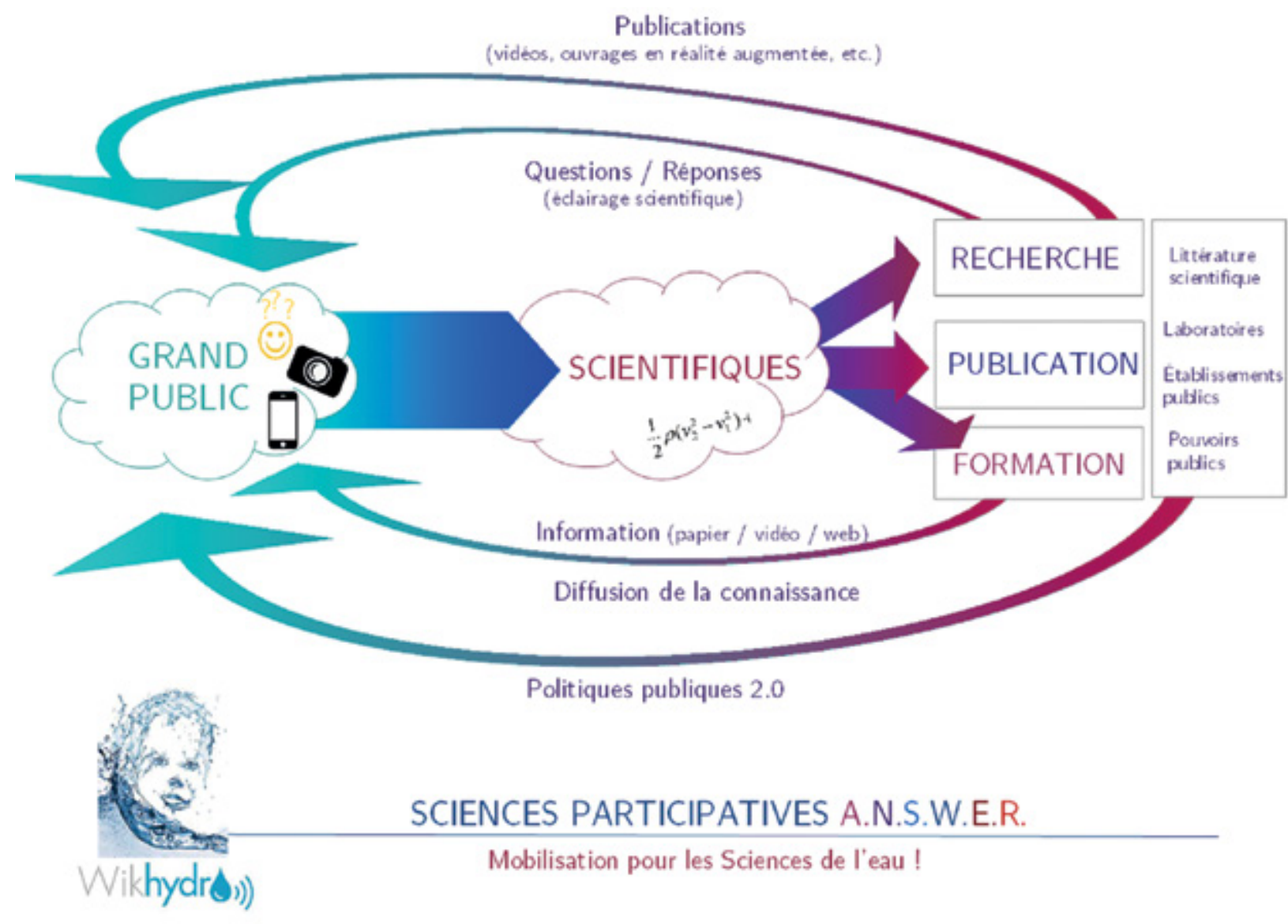

Figure 1 : Échanges entre public et scientifiques.

Ces contributions s'inscrivent dans le contexte du changement climatique et, plus largement, des nombreux changements locaux et globaux.

WIKHYDRO constitue un outil collaboratif de construction de la connaissance et s'accompagne d'une souplesse de publication, modérée a posteriori. La plate-forme contient divers types de documents : images, fichiers pdf et vidéos. Elle incite l'auteur à présenter succinctement et de manière très illustrée ses communications.

\section{III.1. Objectifs de la plate-forme WIKHYDRO}

Outre son ergonomie très souple, la plate-forme collaborative WIKHYDRO a été conçue avec plusieurs objectifs, qui s'attachent à diffuser de la connaissance et à apporter de la plus-value aux organismes et aux personnes. Les avantages sont les suivants :

Pour le public :

- avoir accès à des articles de qualité, écrits par des spécialistes et des experts reconnus dans leurs domaines d'activité et identifiables par leurs productions et leurs publications ; - pouvoir réutiliser les publications, photographies et vidéos, propriétés du ministère, de manière très souple dans leurs activités (avec mention de l'origine du document) ;

- disposer sur le même site évolutif de nombreuses connaissances relatives au domaine de l'eau.

Pour les organismes scientifiques et techniques :

- permettre une diffusion large et rapide des idées nouvelles et des concepts innovants relevant des politiques publiques, en lien avec les avancées de la recherche et de l'innovation ;

- créer un corpus de connaissances commun dans le domaine de l'eau et encourager sa diffusion ;

- thésauriser les connaissances des métiers traditionnels en assurant leur transfert entre générations ;

- mettre à disposition des acteurs de la formation et de l'enseignement des produits actualisés ;

- mettre en valeur des productions des organismes par des renvois sur les sites institutionnels à partir de WIKHYDRO ;

\section{III.2. Les opérations collaboratives dans WIKHYDRO}

Quelques opérations de travail collaboratif sont actuellement en cours : elles regroupent des agents qui travaillent sur les mêmes documents, afin de faire progresser les connaissances sur des sujets bien identifiés : ce sont des espaces ouverts, qui regroupent des contributions collectives. C'est le cas pour ANSWER, pour les drones, le GIS HEDD, le LIDAR...

\section{III.3. Fréquentation de la plate-forme WIKHYDRO}

WIKHYDRO est une plate-forme bien fréquentée et en progression continue, grâce à une animation inlassable et constamment renouvelée qui stimule la communauté de l'eau pour publier de nouveaux articles, des documents, utilisables pour la formation (fiches scientifiques ANSWER), des vidéos de processus, etc... 


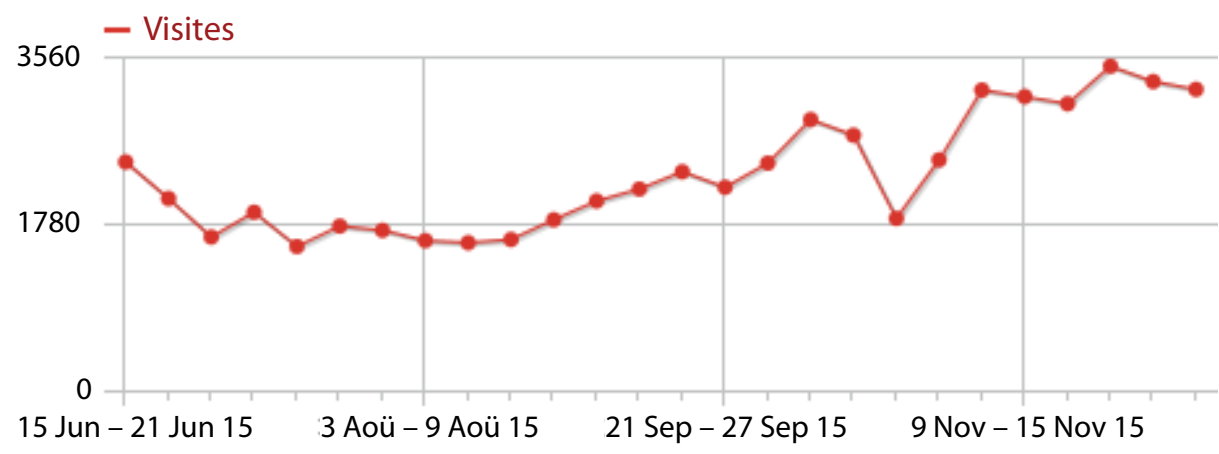

Figure 2 : Fréquentation hebdomadaire du site WIKHYDRO au 10 mars 2015.

\section{RETOMBÉES D'ANSWER}

ANSWER repose donc sur une plate-forme par essence collaborative. Les retombées bénéficient à divers échelons de la société de manière directe :

- le grand public peut participer à l'amélioration des connaissances dans le domaine de l'eau, en complément de la contribution des scientifiques ;

- les scientifiques sont questionnés sur des processus intrigants pouvant donner lieu à des productions techniques, voire des recherches. Les scientifiques du GIS HEDD travaillent actuellement au recensement des solutions analytiques des équations de Navier-Stokes dans les domaines suivants : hydrologie, hydraulique fluviale, hydraulique maritime, hydrogéologie et morphodynamique. Les vidéos réalisées en nature par le public permettent d'illustrer les processus étudiés ;

- les services du ministère et les organismes publics peuvent utiliser ces nouvelles sources d'information pour améliorer les connaissances de leurs agents et réfléchir à la transposition de cette démarche à d'autres thématiques.

\section{QUELQUES PISTES COMPLEMENTAIRES POUR ANSWER}

La finalité d'ANSWER est de mettre en relation les interrogations du grand public avec les recherches des scientifiques qui ont pour objectif d'améliorer les connaissances dans le domaine de l'eau et de trouver des solutions aux grands problèmes de société : santé, risque, aménagement... Cette connexion s'appuie sur des documents audiovisuels :

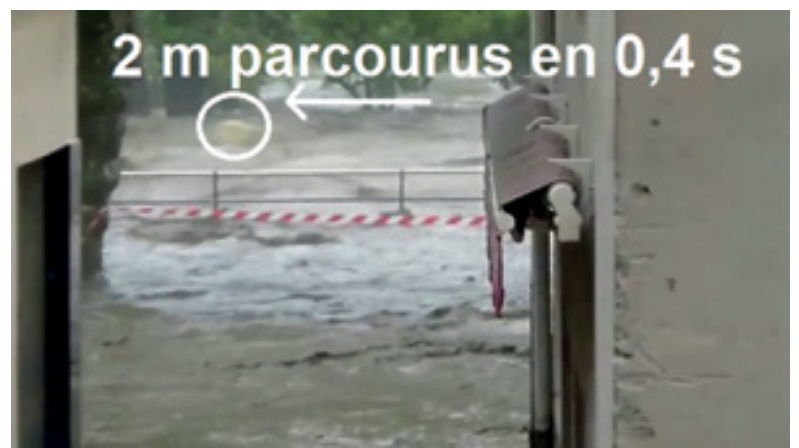

Détermination de la vitesse de l'écoulement photos et vidéos prises souvent sur le vif, selon l'actualité : tempête, crue, pollution ou autres. La démultiplication des smartphones et l'augmentation rapide de la vitesse des transmissions rend techniquement possible cette relation.

Enfin, les citoyens de nos sociétés connectées, reliés par les réseaux sociaux, sont de plus en plus acteurs et participent au débat sociétal en fournissant des informations, en tant qu'individu, groupe (community sourcing) ou en masse (crowd sourcing). Plusieurs opérations de sciences participatives sont déjà en cours dans des domaines de la science où le citoyen, présent partout sur le territoire peut fournir des informations pertinentes et précises et ainsi contribuer à améliorer les connaissances, à prévenir les populations menacées de risques imminents, à surveiller l'environnement.

\section{V.1. Exploitation quantitative des documents produits par le public}

Dans l'état actuel, ANSWER consiste en un simple échange dans les deux sens entre public et scientifiques, mais pourrait très facilement être étendue à une exploitation plus technique des documents récoltés, comme par exemple la quantification des niveaux d'eau pendant une crue ou un épisode de submersion marine, la vitesse en crue ou les temps de submersion de parcelles.

\section{V.1.1. Hydraulique des crues et submersions marines}

Les données produites sont des photos et des vidéos mais peuvent également être des mesures physiques ou des observations de marqueurs simples et accessibles.

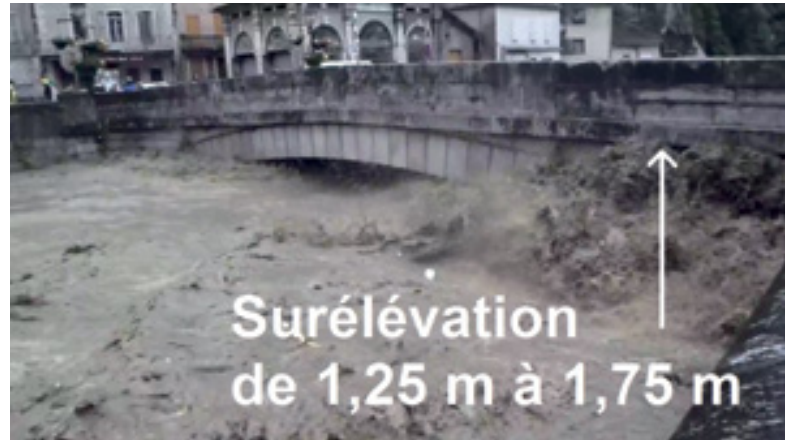

Crue de la Garonne en juin 2013

Figure 3 : Détermination de valeurs de paramètres de l'écoulement (source: O. Payrastre, IFSTTAR). 


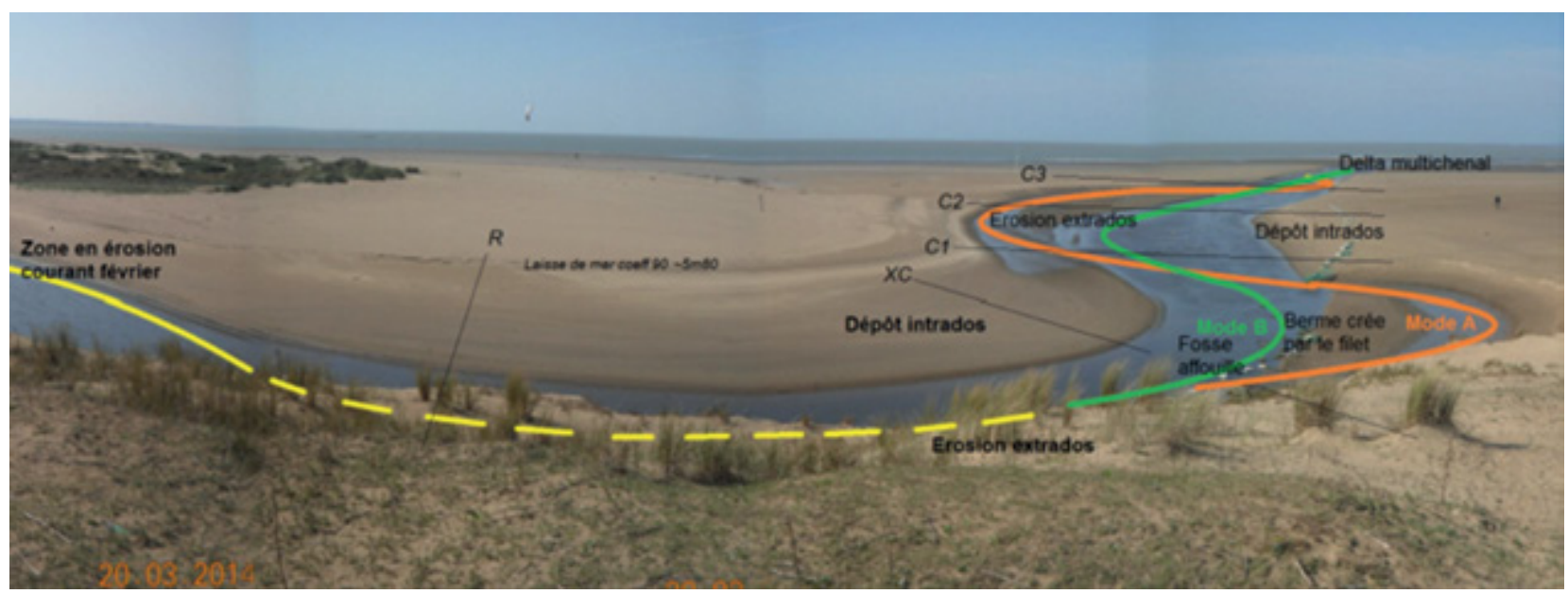

Figure 4 : Suivi des méandres à l'embouchure du Boivre à Saint-Brévin (source DDTM de Loire-Atlantique).

L'intérêt de ces documents réside non seulement dans le témoignage sur l'événement qu'elles représentent: violence du phénomène, hauteur atteinte, dégâts matériels, mais surtout sur la possibilité d'obtenir des données quantitatives à partir de photos/vidéos spécifiques : niveaux atteints facilement identifiables par nivellement a posteriori, vitesse de l'écoulement par déplacement des objets flottants, hauteur et run-up des vagues. Un autre intérêt est de croiser des documents pour approcher certaines valeurs. Vidéos amateur (crues de la Garonne amont en juin 2013).

Les valeurs recherchées sont le niveau d'eau, la vitesse de l'écoulement, le temps de submersion, de ressuyage et les autres données agrégées telles que le débit. À partir de ces retours terrain, il est possible de déterminer les surfaces submergées et leurs évolutions au cours de l'événement.

Ces éléments sont indispensables pour caler les outils de modélisation mais s'avèrent également utiles lors des situations de crise par croisement de scénarios pré-établis et des marqueurs remontés du terrain en temps réel qui permettent de caler le niveau d'intensité des phénomènes.

Dans les cas précédents, les phénomènes, bien que violents, peuvent être observés par plusieurs personnes simultanément. Disposer de témoignages visuels pris en toute sécurité est un élément essentiel pour leur bonne compréhension.

\section{V.1.2. Morphodynamique fluviale ou maritime}

Les photos et vidéos sont souvent pertinentes pour obtenir des informations sur les évolutions des fonds des rivières et des littoraux de manière continue dans l'année (suivi par des bénévoles du trait de côte, d'une instabilité de versant, d'une déviation de chenal, d'une singularité)..

Les photos peuvent être exploitées pour fournir des données complémentaires des mesures existantes par levées terrain ou LIDAR. Les vidéos peuvent également présenter un intérêt pour les processus instantanés violents : rupture d'un pan de falaise, de berges entraînant la destruction de maisons, submersions, ouverture de brèches dans les digues, rupture d'ouvrages : barrages, seuils... qui ont des conséquences sur la sécurité des personnes et des biens.

\section{PREMIERS RÉSULTATS}

L'opération ANSWER a été construite sur ces principes et les premiers résultats obtenus montrent qu'elle suscite l'intérêt d'un public averti, impliqué dans le domaine de l'eau.

Il ne faut pas non plus occulter les faiblesses inhérentes à la nature de l'opération elle-même. La première tient aux documents émanant du grand public qui varient fortement en fonction des connaissances du sujet par l'individu. Il est possible que la grande majorité des documents produits ne présente que peu d'intérêt pour les scientifiques. Cependant, on peut trouver dans la masse des productions, un certain nombre de pépites qui peuvent interpeller les scientifiques et générer de la recherche. Le rayonnement du site pourrait facilement être amélioré par une communication extensive.

Ce type d'opérations de sciences participatives est appelée à se développer pour plusieurs raisons : la diffusion de nouvelles technologies, la production de données en masse (big data) et leur exploitation avec de nouveaux outils très performants, l'impossibilité des pouvoirs publics de continuer à être les principaux producteurs de données dans tous les domaines, les nouvelles richesses qui peuvent être générées à partir de l'exploitation de ces grandes bases de données, l'implication de plus en plus importante des citoyens qui détiennent une véritable expertise sur leur territoire et qui participent au débat public.

L'initiative ANSWER répond à la plupart de ces critères et contribue à créer une véritable dynamique collaborative dans le domaine de l'eau.

\section{REFERENCES}

NiELSEN M.. (2012) — Reinventing Discovery - The New Area of Networked Science. Princeton University Press.

Boeuf G., Y. Marie-Allain, M. Bouvier (2012) — L'apport des sciences participatives à la connaissance de la biodiversité. Rapport remis à la Ministre de l'écologie. 30 pages 